\title{
A THEOREM ON INFINITE PRODUCTS OF EIGENVALUES OF STURM-LIOUVILLE TYPE OPERATORS
}

\section{S. LEVIT AND U. SMILANSKY}

\begin{abstract}
Infinite products of ratios of eigenvalues of Sturm-Liouville operators are expressed in a closed form in terms of corresponding solutions of initial-value problems.
\end{abstract}

Introduction. Gaussian path integrals are defined by

$$
I=\int_{\text {paths }} D[\vec{\eta}(t)] \exp [i S[\vec{\eta}(t)]]
$$

where

$$
S[\vec{\eta}(t)]=\int_{0}^{T}(\vec{\eta}(t) \cdot \Lambda(t) \vec{\eta}(t)) d t,
$$

and $\vec{\eta}(t)$ are the $N$ dimensional paths satisfying the end conditons $\vec{\eta}(0)=$ $\vec{\eta}(T)=0 . \Lambda(t)$ is a differential operator of second order in $(d / d t)$ acting on the space of paths. Such path integrals are often encountered in physical problems, e.g. the formulation of quantum mechanics [1].

The integral (1) can be evaluated in terms of the infinite product of the eigenvalues of $\Lambda(t)$ [2], [3]. In this note we present a theorem which enables one to express these infinite products in a closed form.

The theorem will be presented with a detailed proof for the simple case of a one dimensional problem $(N=1)$. The extension to $N>1$ will be formulated but a detailed proof will not be supplied since it follows the same lines as for $N=1$.

THEOREM 1. Let the differential operator

$$
\Lambda(\alpha ; t)=(d / d t)(p(t) d / d t)+\alpha q(t)
$$

be defined for $0 \leqslant t \leqslant T, 0 \leqslant \alpha \leqslant 1$, where $p(t)$ and $q(t)$ are smooth functions of $t, p(t)>p_{0}>0$.

Let $\lambda_{k}(\alpha)$ and $U^{(k)}(\alpha ; t)$ be the eigenvalues and eigenfunctions for the boundary-value problem

(4) $\Lambda(\alpha ; t) U(\alpha ; t)+\lambda(\alpha) U(\alpha ; t)=0, \quad U(\alpha ; 0)=U(\alpha ; T)=0$.

Let $y(\alpha ; t)$ be the solution of the initial-value problem

Received by the editors January 20, 1976.

AMS (MOS) subject classifications (1970). Primary 34B25; Secondary 34E10.

Key words and phrases. Products of eigenvalues of Sturm-Liouville operators.

○ American Mathematical Society 1977 


$$
\Lambda(\alpha ; t) y(\alpha ; t)=0, \quad y(\alpha ; 0)=0, \quad(d / d t) y(\alpha ; 0)=1
$$

Then, for every $\alpha$

$$
\left|\prod_{k=1}^{\infty} \frac{\lambda_{k}(\alpha)}{\lambda_{k}(0)}\right|=\left|\frac{y(\alpha ; T)}{y(0 ; T)}\right| .
$$

Proof. The following points should be observed:

(a) For all $k, \lambda_{k}(0)>0$ and for large $k$,

$$
\lambda_{k}(0) \cong\left[\pi / \int_{0}^{T} \frac{d t}{\sqrt{p(t)}}\right]^{2} \cdot k^{2},[4]
$$

(b) The functions $\lambda_{k}(\alpha), U^{(k)}(\alpha ; t)$ and $y(\alpha ; t)$ are analytic in $\alpha$ [5].

(c) For large $k,\left|\lambda_{k}(\alpha)-\lambda_{k}(0)\right| \leqslant \alpha \cdot M$ [4] with $M$ independent of $\alpha$ or $k$. Hence the product on the left-hand side of (6) converges uniformly for all finite values of $\alpha$.

(d) There is a finite number of nonpositive eigenvalues for a given $\alpha$ [6]. Hence the product on the left-hand side of (6) vanishes only at a finite number of $\alpha$ values, in the interval $0 \leqslant \alpha \leqslant 1$.

A necessary and sufficient condition for $y(\alpha ; T)$ to vanish is that one of the $\lambda_{k}(\alpha)$ vanishes. Hence the two sides of equation (6) vanish simultaneously.

Consider now the functions:

$$
\begin{gathered}
f(\alpha)=\prod_{k=1}^{\infty} \frac{\lambda_{k}(\alpha)}{\lambda_{k}(0)}, \\
\tilde{f}(\alpha)=y(\alpha ; T) / y(0 ; T) .
\end{gathered}
$$

Since by construction $f(0)=\tilde{f}(0)=1$, the theorem will be proved once it is demonstrated that $(d / d \alpha) f(\alpha) / f(\alpha)=(d / d \alpha) \tilde{f}(\alpha) / \tilde{f}(\alpha)$, for all $\alpha$ satisfying $f(\alpha) \neq 0$.

By virtue of (c) one can differentiate the left-hand side of equation (7) as if it were a finite product and get

$$
\frac{d}{d \alpha} f(\alpha) / f(\alpha)=\sum_{k=1}^{\infty} \frac{d \lambda_{k}(\alpha)}{d \alpha} \frac{1}{\lambda_{k}(\alpha)}
$$

It can easily be shown that

$$
\frac{d \lambda_{k}(\alpha)}{d \alpha}=-\int_{0}^{T}\left[U^{(k)}(\alpha ; t)\right]^{2} q(t) d t,
$$

where $U^{(k)}(\alpha ; t)$ are the solutions of (4) subject to the normalization

$$
\int_{0}^{T}\left[U^{(k)}(\alpha ; t)\right]^{2} d t=1
$$


Since the integral in (10) is bounded by $\operatorname{Max}|q(t)|$ and because of (c), the series on the right-hand side of (9) converges uniformly and the summation and integration operations could be interchanged to yield

$$
\frac{d}{d \alpha} f(\alpha) / f(\alpha)=\int_{0}^{T} G_{\lambda=0}(t, t, \alpha) q(t) d t
$$

with

$$
G_{\lambda}\left(t, t^{\prime}, \alpha\right)=\sum_{k=1}^{\infty} \frac{U^{(k)}(\alpha ; t) U^{(k)}\left(\alpha ; t^{\prime}\right)}{\lambda(\alpha)-\lambda_{k}(\alpha)},
$$

which is the spectral representation of Green's function for the boundaryvalue problem (4).

Returning to the initial value problem (5) and differentiating it with respect to $\alpha$, one gets that $z(\alpha ; t) \equiv d y(\alpha ; t) / d \alpha$ is a solution of the initial-value problem

$$
\Lambda(\alpha ; t) z(\alpha ; t)=-q(t) y(\alpha ; t), \quad z(\alpha ; 0)=0, \quad(d / d t) z(\alpha ; 0)=0 .
$$

The solution of (14) is expressed in terms of the solution $y(\alpha ; t)$ of $(5)$ and the solution $\tilde{y}(\alpha ; t)$ of the adjoint problem

$$
\Lambda(\alpha ; t) \tilde{y}(\alpha ; t)=0, \tilde{y}(\alpha ; T)=0, \quad(d / d t) \tilde{y}(\alpha ; T)=1 .
$$

Applying the method of variation of the parameters, one can prove that the solution of (14) is given by

where

$$
\begin{aligned}
& z(\alpha ; t)=\left[y(\alpha ; t) \int_{0}^{t} y\left(\alpha ; t^{\prime}\right) \tilde{y}\left(\alpha ; t^{\prime}\right) q\left(t^{\prime}\right) d t^{\prime}\right. \\
& \left.-\tilde{y}(\alpha ; t) \int_{0}^{t} y\left(\alpha ; t^{\prime}\right) y\left(\alpha ; t^{\prime}\right) q\left(t^{\prime}\right) d t^{\prime}\right] / W,
\end{aligned}
$$

$$
W=p(t)[y d \tilde{y} / d t-\tilde{y} d y / d t]=\text { const. }
$$

Hence

$$
\begin{aligned}
z(\alpha ; T) & =\frac{d y(\alpha ; T)}{d \alpha}=y(\alpha ; T) \int_{0}^{T} \tilde{y}(\alpha ; t) y(\alpha ; t) q(t) d t / W \\
& =y(\alpha ; T) \int_{0}^{T} G_{\lambda=0}(t, t, \alpha) q(t) d t
\end{aligned}
$$

where $G_{\lambda}\left(t, t^{\prime}, \alpha\right)$ is again Green's function for the boundary-value problem (4), expressed in terms of the solutions of the initial-value problem (5) and its adjoint (15). A comparison of (18) with (12) completes the proof.

We now turn to the generalization of Theorem 1 to the $N$ dimensional case. The functions $p(t)$ and $q(t)$ of (3) are now replaced by the $N \times N$ symmetric matrices $P(t)$ and $Q(t) . P(t)$ is required to be positive definite for all $0 \leqslant t \leqslant T$. The $N$ dimensional vectors on which $\Lambda(\alpha ; t)$ acts are denoted by $\vec{U}(\alpha ; t) \equiv\left(U_{1}(\alpha ; t), \ldots, U_{N}(\alpha ; t)\right)$.

THEOREM 2. Let a differential operator 


$$
\Lambda(\alpha ; t)=(d / d t)(P(t) d / d t)+\alpha Q(t)
$$

be defined for $0 \leqslant t \leqslant T, 0 \leqslant \alpha \leqslant 1$, with $P(t)$ and $Q(t)$ as discussed above.

Let $\lambda_{k}(\alpha)$ and $\vec{U}^{(k)}(\alpha ; t)$ be the eigenvalues and eigenfunctions for the boundary-value problem

$$
\Lambda(\alpha ; t) \vec{U}(\alpha ; t)+\lambda(\alpha) \vec{U}(\alpha ; t)=0, \quad \vec{U}(\alpha ; 0)=\vec{U}(\alpha ; T)=0
$$

Let $\vec{y}^{(j)}(\alpha ; t)(j=1, \ldots, N)$ be the $N$ independent solutions of the initialvalue problem

$$
\Lambda(\alpha ; t) \vec{y}^{(j)}(\alpha ; t)=0, \quad \vec{y}^{(j)}(\alpha ; 0)=0, \quad(d / d t) y_{i}^{(j)}(\alpha ; 0)=\delta_{i, j} .
$$

Let $D(\alpha)$ be

$$
D(\alpha)=\operatorname{det}\left[y_{i}^{(j)}(\alpha ; T)\right]
$$

Then, for every $\alpha$

$$
\left|\prod_{k=1}^{\infty} \frac{\lambda_{k}(\alpha)}{\lambda_{k}(0)}\right|=\left|\frac{D(\alpha)}{D(0)}\right|
$$

OUTLINE OF THE PROOF. The proof of Theorem 2 is again based on evaluating the logarithmic derivative of the two sides of (23). Once again it is shown that the logarithmic derivative can be expanded in the form

$$
\frac{d f(\alpha)}{d \alpha} / f(\alpha)=\sum_{j, i=b}^{N} \int_{0}^{T} d t G_{j, i}^{(\lambda=0)}(t, t, \alpha) Q_{i j}(t)=\frac{d \tilde{f}(\alpha)}{d \alpha} / \tilde{f}(\alpha)
$$

where $f(\alpha)$ and $\tilde{f}(\alpha)$ denote the functions of $\alpha$ on the left- and right-hand sides of equation (23) respectively. The Green's "function" is a matrix $G_{i, j}^{(\lambda)}\left(t, t^{\prime}, \alpha\right)$. In proving (24) use is made of the two equivalent methods to express the Green's function, the spectral representation and the representation by means of the $2 N$ independent solutions of equation (21) and its adjoint.

Acknowledgement. We are indebted to Dr. H. Dym of the Department of Mathematics of the Weizmann Institute for valuable comments and suggestions.

\section{REFERENCES}

1. R. P. Feynman and A. R. Hibbs, Quantum mechanics and path integrals, McGraw-Hill, New York, 1965.

I. M. Gel'fand and A. M. Yaglom, J. Math. Phys.1 (1960), p. 48.

2. M. C. Gutzwiller, J. Math. Phys. 8 (1967), p. 1979.

3. S. Levit and U. Smilansky, Ann. Phys. 103 (1977), p. 198.

4. P. M. Morse and H. Feshbach, Methods of theoretical physics. I, McGraw-Hill, New York, 1953, pp. 1-997. MR 15, 583.

5. H. Poincaré, Acta Math. 4 (1884), p. 213.

6. M. Morse, The calculus of variations in the large, Amer. Math. Soc. Colloq. Publ., vol. 18, Amer. Math. Soc., Providence, R. I., 1934; reprint 1966.

Department of Nuclear Physics, Weizmann Institute of Science, Rehovot, Israel 